\title{
ON A CLASS OF QI-RINGS
}

\section{by S. K. JAIN, S. R. LÓPEZ-PERMOUTH† and SURJEET SINGH}

\author{
(Received 14 August, 1990)
}

0. Introduction. The concept of weak relative-injectivity of modules was introduced originally in [10], where it is shown that a semiperfect ring $R$ is such that every cyclic right module is embeddable essentially in a projective right $R$-module if and only if $R$ is right artinian and every indecomposable projective right $R$-module is uniform and weakly $R$-injective. We show that in the above characterization the requirement that indecomposable projective right $R$-modules be uniform is superfluous (Theorem 1.11). In this paper we further the study of weak relative-injectivity by considering the class of rings for which every right module is weakly injective relative to every finitely generated right module. We refer to such rings as right weakly-semisimple rings. The class of right weakly-semisimple rings includes properly all semisimple rings and is a subclass of the class of right QI-rings. A ring $R$ is said to be a right QI-ring if every quasi-injective right $R$-module is injective. QI-rings have been studied in [2], [3], [4], [6], [7], [8], [11], among others.

In [4], Boyle characterizes right QI-rings as being those right noetherian rings for which every uniform cyclic right module is strongly prime. In contrast, we show that a ring $R$ is right weakly-semisimple if and only if $R$ is right noetherian and every finitely generated uniform right $R$-module is compressible (Theorem 2.5). While it is not clear at this time if there exist any non-weakly-semisimple right QI-rings, we show that a two-sided noetherian and hereditary ring is right weakly-semisimple if and only if it is right QI (Theorem 3.1). This implies that an example of a right QI-ring which is not weakly-semisimple will necessarily be either a counter-example to Boyle's conjecture or a right QI-ring which is not left QI.

Since a weakly $R^{2}$-injective semiprime right Goldie ring must be left Goldie, we are able to apply some results of Kosler [11] to get that a right weakly-semisimple ring with restricted right minimum condition is left weakly-semisimple (Theorem 3.4).

Throughout this paper all rings have 1 and all modules are unital right unless otherwise stated. The injective hull of the right $R$-module $M$ is denoted by $E\left(M_{R}\right)$ or simply $E(M)$ if there is no ambiguity.

\section{Weak relative injectivity.}

1.1. Definition. Given two right $R$-modules $M$ and $N$, we say that $M$ is weakly $N$-injective (wNi) if for every homomorphism $f: N \rightarrow E(M)$ there exists a monomorphism $\sigma: M \rightarrow E(M)$ and a homomorphism $\hat{f}: N \rightarrow M$ such that $f=\sigma \hat{f}$. Notice that this concept generalizes that of $M$ being $N$-injective in which case we require $\sigma$ to be the inclusion map.

1.2. Lemma. Given two right modules $M$ and $N$, the following statements are equivalent:

(i) $M$ is weakly $N$-injective;

(ii) for every submodule $K$ of $N, M$ is weakly $N / K$-injective;

† The second author wishes to acknowledge the partial support of the Ohio University Research Committee under grant \#825. 
(iii) for every submodule $K$ of $N$, and for every monomorphism $h: N / K \rightarrow E(M)$, there exists a monomorphism $\sigma: M \rightarrow E(M)$ and a monomorphism $\hat{h}: N / K \rightarrow M$ such that $\hat{h}=\sigma h$.

Proof. Straightforward.

When we say that $M$ is weakly $N$-injective, we are saying more than "every quotient of $N$ which is embeddable in $E(M)$ is embeddable in $M$." This is the subject of our next lemma.

1.3. Lemma. Given two right modules $M$ and $N, M$ is weakly $N$-injective if and only if for every submodule $Q$ of $N$ and for every monomorphism $\sigma: N / Q \rightarrow E(M)$ :

(i) there exists a monomorphism $\sigma^{\prime}: N / Q \rightarrow M$, and

(ii) for every complement $K$ of $\sigma^{\prime}(N / Q)$ in $M$ there exists $K^{\prime} \subset E(M)$ such that $K^{\prime} \cap \sigma(N / Q)=0$ and $K^{\prime} \simeq K$.

Proof. Let $\sigma: N / Q \rightarrow E(M)$ be a monomorphism. By Lemma 1.2(iii), there exist monomorphisms $\alpha: M \rightarrow E(M)$ and $\sigma^{\prime}: N / Q \rightarrow M$ such that $\sigma=\alpha \sigma^{\prime}$. Thus (i) holds. Let $K$ be a complement of $\sigma^{\prime}(N / Q)$ in $M$; then $K^{\prime}=\alpha(K)$ is isomorphic to $K$ and independent from $\sigma(N / Q)$ proving that (ii) is also necessary. Conversely, let us assume that (i) and (ii) hold and let $\sigma: N / Q \rightarrow E(M)$ be a monomorphism. By (i) there exists $\sigma^{\prime}: N / Q \rightarrow M$. Let $K$ be a complement of $\sigma^{\prime}(N / Q)$ in $M$. Using (ii), we get a monomorphism $\alpha: \sigma^{\prime}(N / Q) \oplus K \rightarrow E(M)$. Since $\sigma^{\prime}(N / Q) \oplus K \subset^{\prime} M$, we may extend $\alpha$ to a monomorphism $\beta: M \rightarrow E(M)$. It is straightforward that $\beta \sigma^{\prime}=\sigma$. Using Lemma 1.2 (iii) gives us that $M$ is weakly $N$-injective.

1.4. Corollary. For a uniform module $M, M$ is weakly $N$-injective if and only if every quotient of $N$ which is embeddable in $E(M)$ is embeddable in $M$.

Proof. Obvious.

1.5. Remark. Let $n \in \mathbb{Z}^{+}$. A module $M$ is weakly $R^{n}$-injective if and only if for every $x_{1}, x_{2}, \ldots, x_{n} \in E(M)$ there exists a submodule $X$ of $E(M)$ such that $X$ is isomorphic to $M$ and $x_{1}, x_{2}, \ldots, x_{n} \in X$.

Proof. Obvious.

A right module $M$ is injective if and only if it is weakly $N$-injective for every right module $N$. With this in mind, we make the following definition.

1.6. Definition. A right $R$-module $M$ is weakly-injective if it is weakly $R^{n}$-injective for all $n \in \mathbb{Z}^{+}$.

In view of Lemma 1.2, $M$ is weakly-injective if and only if it is weakly $N$-injective for every finitely generated right module $N$.

1.7. Proposition. Let $M, N$ and $P$ be right modules.

(a) If $M$ and $N$ are weakly $P$-injective, then so is $M \oplus N$.

(b) If $M$ is weakly $P$-injective and $M \subset^{\prime} N$, then $N$ is weakly $P$-injective.

(c) It is possible for $M \oplus N$ to be weakly P-injective while $M$ is not weakly $P$-injective.

(d) It is possible for $M \subset^{\prime} N, N$ weakly $P$-injective but $M$ not weakly $P$-injective.

Proof. See [10]. 
1.8. Lemma. Let $U$ be $M$-injective with $M$ weakly $R$-injective, then $U$ is $E(M)$ injective.

Proof. If $U$ is not $E(M)$-injective, then by Zorn's lemma there exists a submodule $A$ of $E(M)$ and a homomorphism $f: A \rightarrow U$ which cannot be extended to any $f^{\prime}: B \rightarrow U$ with $B$ a submodule of $E(M)$ containing $A$ properly. Let $b \in E(M) / A$. Notice that $A \subset^{\prime} E(M)$; so $C=b R \cap A \neq 0$. Let $f_{1}: C \rightarrow U$ be the restriction of $f$ to $C$. As $M$ is weakly $R$-injective, $b R$ embeds in $M$. Therefore, $U$ is $b R$-injective and $f_{1}$ extends to $g: b R \rightarrow U$. Define $f^{\prime}: A+b R \rightarrow U$ by $f^{\prime}(a+b r)=f(a)+g(b r)$, whenever $a \in A, r \in R$. Since $f^{\prime}$ extends $f$, this is a contradiction. Therefore, $U$ is $E(M)$-injective.

1.9. Lemma. A weakly $R$-injective quasi-injective right module $M$ is injective.

Proof. Take $U=M$ in Lemma 1.8 .

1.10. Proposition. Let $R$ be an artinian ring and $P=e R$ be a projective indecomposable module which is weakly $R$-injective. Then $P$ is uniform.

Proof. We aim to show that $E=E(P)$ is indecomposable. Let $E=A \oplus B, A \neq 0, A$ indecomposable. Let $a_{0} \in A, a_{0} \neq 0$. By weak $R$-injectivity there exists $a_{1}+b_{1} \in E$ such that $r\left(a_{1}+b_{1}\right)=r\left(a_{1}\right) \cap r\left(b_{1}\right)=(1-e) R=0$ and $a_{0} \in\left(a_{1}+b_{1}\right) R$. So, actually $a_{0} R \subseteq a_{1} R$. Inductively $a_{0} R \subseteq a_{1} R \subseteq \ldots \subseteq\left(a_{n}+b_{n}\right) R \simeq e R$, where $n$ is larger than or equal to the composition length of $e R$. Thus, there exists $i$ such that $a_{i} R=a_{i+1} R$, i.e. there exists $x \in r\left(b_{i+1}\right)$ such that $a_{i}=a_{i+1} x$, and there exists $y \in R$ such that $a_{i} y=a_{i+1}$. It follows that $a_{i+1} x y=a_{i+1}$; hence $1-x y \in r\left(a_{i+1}\right)$. Since $x y \in r\left(b_{i+1}\right)$, we get that $r\left(a_{i+1}\right)+r\left(b_{i+1}\right)=R$.

Now $r\left(a_{i+1}\right)=(1-e) R \oplus e R \cap r\left(a_{i+1}\right)$ and $r\left(b_{i+1}\right)=(1-e) R \oplus e R \cap r\left(b_{i+1}\right)$. It follows that $\left(e R \cap r\left(a_{i+1}\right)\right)+\left(e R \cap r\left(b_{i+1}\right)\right)=e R$. Since $e R$ is local, this implies that $r\left(a_{i+1}\right)=R$ or $r\left(b_{i+1}\right)=R$. However, $a_{i+1} \neq 0$; hence $r\left(b_{i+1}\right)=R$ and consequently $b_{i+1}=0$. Thus $e R \simeq a_{i+1} R \subseteq A$, proving that $e R$ is uniform.

Proposition 1.10 yields the following theorem which sharpens the characterization of right CEP-rings in [10].

1.11. Theorem. A semiperfect ring $R$ is right CEP if and only if $R$ is right artinian and every projective indecomposable right $R$-module is weakly $R$-injective.

1.12. Lemma. A cyclic right module $M$ is weakly-injective if and only if it is weakly $R^{2}$-injective.

Proof. One implication is trivial. Suppose that $M$ is weakly $R^{2}$-injective and let us proceed by induction. Let $M$ be weakly $R^{n-1}$-injective and let $x_{1}, x_{2}, \ldots, x_{n-1} \in E(M)$. By the inductive hypothesis, there exists $x R \subseteq E(M)$ such that $x_{1}, \ldots, x_{n-1} \in x R$. By the weak $R^{2}$-injectivity of $M$, there exists $X \simeq M$ such that $x, x_{n} \in X$. Hence $x_{1}, x_{2}, \ldots, x_{n} \in$ $X$, concluding our proof.

1.13. ExAmples. (i) $A$ ring $R$ is quasi-Frobenius if and only if it is right artinian and right weakly-injective.

(ii) $A$ domain $R$ is right weakly $R$-injective if and only if it is right Ore and right weakly-injective if and only if it is two-sided Ore.

(iii) Every semiprime right and left noetherian ring is weakly-injective. 
(iv) $A$ ring $R$ is right self-injective if and only if it is right continuous and right weakly-injective.

(v) A semiprime right weakly-injective right Goldie ring is left Goldie.

Proof. (i) One implication is trivial. Let $R$ be right artinian and weakly-injective. Let $x \in E(R)$. By weak-injectivity there exist $X \subseteq E(R), X \simeq R$, such that $1, x \in X$. Then $R \subseteq X$ and $X$ is right artinian and isomorphic to $R$. We conclude that $R=X$ and, therefore, $x \in R$. So $R=E(R)$ is quasi-Frobenius.

(ii) Let $R$ be a domain. The injective hull of $R$ is its Utumi ring of quotients $Q$. Assume that $R$ is right Ore then $Q$ is the classical right ring of quotients of $R$, a division ring. The result is obvious. On the other hand, if $R$ is right weakly $R$-injective, for every $q \in Q$ there exists $q^{\prime} \in Q$ with $r\left(q^{\prime}\right)=0$ and $r \in R$ such that $q=q^{\prime} r$. This implies that $Q$ is again a domain and, therefore, being self-injective, a division ring. Thus, $R$ is right Ore. If $R$ is two-sided Ore, then $Q$ is both a right and a left ring of quotients for $R$. Let $q_{1}, q_{2} \in Q$; then there exists $r_{1}, r_{2}, s \in R$ such that $q_{1}=s^{-1} r_{1}, q_{2}=s^{-1} r_{2}$. Since $r\left(s^{-1}\right)=$ $0, q_{1}, q_{2} \in s^{-1} R$, using Lemma 1.12 we conclude that $R$ is right weakly-injective. The converse follows from $(\mathrm{y})$.

(iii) Let $R$ be a right and left noetherian semiprime ring. Then $E\left(R_{R}\right)=E\left({ }_{R} R\right)=$ $r \cdot Q_{\mathrm{cl}}(R)=l \cdot Q_{\mathrm{cl}}(R)$ where $r \cdot Q_{\mathrm{cl}}(R)$ and $l \cdot Q_{\mathrm{cl}}(R)$, respectively, denote right and left classical ring of quotients of $R$. Let $q_{1}, q_{2} \in Q$; then there exists $r_{1}, r_{2} \in R, s \in R /\{0\}$ such that $q_{1}=s^{-1} r_{1}, q_{2}=s^{-1} r_{2}$. We conclude that $R$ is weakly-injective by using Lemma 1.12 .

(iv) While one implication is trivial, the converse requires only a weak version of condition $\mathrm{C}_{2}$ in the definition of continuity [13], namely "a submodule which is isomorphic to a summand must be closed". Let $R$ be weakly-injective satisfying the above condition. Let $x \in E(R)$. There exists $X \subseteq E(R)$ which is isomorphic to $R$ and contains 1 and $x$. Since the submodule $R$ of $X$ is isomorphic to $X$ and, therefore, $X$ also satisfies the above hypothesis, $R$ is closed in $X$. On the other hand, $R+x R \subseteq E(R)$ hence $R+x R$ is an essential extension of $R$ in $X$. We conclude that $x \in R$, and so $R=E(R)$ is self-injective.

(v) Let $R$ be a semiprime right Goldie ring. Then the injective hull of $R$ is its complete ring of right quotients $Q$. Assume $R$ is weakly-injective and let $q \in Q$. There exists $q^{\prime} \in Q$ such that $r\left(q^{\prime}\right)=0$ with $1, q \in q^{\prime} R$. It follows that $q^{\prime}=r^{-1}$ for some $r \in R$ and there exists $s \in R$ such that $q=r^{-1} s$. Therefore, $Q$ is a left ring of quotients for $R$ and hence $R$ is left Goldie.

2. Rings all of whose modules are weakly-injective. We recall that a right module $M$ is weakly-injective if it is weakly $R^{n}$-injective for all $n \in \mathbb{Z}^{+}$(Definition 1.6). Remark 1.2 implies that $M$ is weakly-injective if and only if it is weakly $N$-injective for every finitely generated right $R$-module $N$.

2.1. Definition. A ring $R$ is said to be right weakly-semisimple if every right module $M$ is weakly-injective.

\subsection{LeMMA. A right weakly-semisimple ring is right $Q I$.}

Proof. Obvious from Lemma 1.19.

Before introducing our next lemma, remember that a right module $M$ is said to be compressible if for all nonzero $N \subseteq M$ there exists a monomorphism from $M$ into $N$ [9]. 
2.3. Lемма. Given an indecomposable injective right module $E$, the following statements are equivalent:

(i) every submodule of $E$ is weakly-injective;

(ii) every cyclic submodule of $E$ is weakly-injective;

(iii) every finitely generated submodule of $E$ is compressible.

Proof. Clearly (i) implies (ii). Assume (ii) and let $M$ be a finitely generated submodule of $E$ and $N$ a non-zero submodule of $M$. Let $x \in N, x \neq 0$. Since $x R$ is weakly-injective, $M$ is embeddable in $x R$ and hence in $N$. Let us now assume (iii). Let $N$ be an arbitrary non-zero submodule of $E$. Suppose $x \in N, x \neq 0$. If $x_{1}, x_{2}, \ldots, x_{n} \in$ $E(N)=E$ by the compressibility of $M$, where $M=\sum_{i=1}^{n} x_{i} R$, there exists an embedding $\sigma$ of $M$ into $x R \subseteq N$. Using Corollary 1.4 , we conclude that $N$ is weakly-injective.

2.4. LEMMA. A right noetherian ring $R$ over which every finitely generated $R$-module is weakly-injective must be weakly-semisimple.

Proof. Let $M$ be a right $R$-module and $x_{1}, x_{2}, \ldots, x_{n} \in E(M)$. Let $K=\sum_{i=1}^{n} x_{i} R$. It follows that $E(M)=E(K) \oplus L$ for some submodule $L$ of $E(M)$, and $E(K)$ has finite Goldie dimension. Therefore, $M \cap E(K)$, being essential in $E(K)$, also has finite Goldie dimension. Let $N$ be a finite direct sum of uniform cyclics which is essential in $M \cap E(K)$. By hypothesis $N$ is weakly-injective. Thus there exists an automorphism $\sigma: E(K) \rightarrow E(K)$ with $K \subseteq \sigma(N) \subseteq \sigma(M \cap E(K))$. Clearly $\sigma$ extends to an automorphism $\eta$ of $E(M)$ which is the identity on $L$. This automorphism satisfies that $K \subseteq \eta(M)$, concluding our proof.

2.5. THEOREM. The following conditions on a ring $R$ are equivalent:

(i) $R$ is right weakly-semisimple;

(ii) every finitely generated right $R$-module is weakly-injective and $R$ is right noetherian;

(iii) every cyclic right $R$-module is weakly $R^{2}$-injective and $R$ is right noetherian;

(iv) every uniform cyclic right $R$-module is weakly $R^{2}$-injective and $R$ is right noetherian;

(v) every finitely generated uniform right $R$-module is compressible and $R$ is right noetherian.

Proof. From Lemma 2.2 and the fact that every right QI-ring is right noetherian, it follows that (i) implies (ii). Clearly (ii) implies (iii) and (iii) implies (iv). The implication (ii) $\Rightarrow$ (i) is Lemma 2.4. Since every finitely generated module over a right noetherian ring contains essentially a finite direct sum of cyclics, using Proposition 1.7(a) and (b) and Lemma 2.3, we obtain that (iv) implies (ii). The equivalence of (iv) and (v) follows from Lemma 2.3 .

2.6. REMARK. Theorem 2.5 is specially interesting when compared to a result by Boyle which characterizes right QI-rings as being those right noetherian rings for which every uniform cyclic is strongly prime [4] in the following sense. A module $M$ is strongly prime [1] if it satisfies one of the equivalent conditions:

(i) $M$ is contained in every quasi-injective submodule of its injective hull, or 
(ii) for all $x, y \in M$, there exists $r_{1}, r_{2}, \ldots, r_{n} \in R$ such that $r(x)=$ $r\left(y r_{1}, y r_{2}, \ldots, y r_{n}\right)$.

It can be shown easily that every compressible module is strongly prime.

In relation to Theorem 2.5 and Remark 2.6, it would be interesting to characterize right noetherian rings in which every cyclic right $R$-module is weakly $R$-injective and right noetherian rings in which every cyclic uniform right $R$-module is compressible. Also, could the condition of $R$ being right noetherian be removed from any of the equivalent statements (ii) through (v) in Theorem 2.5? While it does not seem likely, we do not have a counter-example.

3. The noetherian hereditary case. Every right QI-ring is a right noetherian right V-ring. Also, it is well known [3] that for a (two-sided) noetherian hereditary ring $R$, the following conditions are equivalent:

(i) $R$ is a right $V$-ring;

(ii) $R$ is a left $\mathrm{V}$-ring;

(iii) $R$ is a right QI-ring;

(iv) $R$ is a left QI-ring.

Our next theorem extends this list of equivalent statements to include right and left weakly-semisimple.

3.1. ThEOREM. Let $R$ be a hereditary noetherian ring. Then the following are equivalent:

(i) $R$ is a right $Q I-$ ring;

(ii) $R$ is a right weakly-semisimple ring;

(iii) $R$ is a left weakly-semisimple ring.

Proof. It suffices to show that (i) implies (ii). Without losing generality, we may assume that $R$ is simple [12]. Reasoning as in [2, Theorem 5], we may also assume that $R$ is a right (and left) Ore domain. Let $M$ be a finitely generated uniform right $R$-module. Since every finitely generated right module over a hereditary noetherian prime ring is a direct sum of a projective and a torsion module [5, Theorem 2.1], $M$ is either projective or torsion. If $M$ is projective, then, being uniform, $M$ must be embeddable in $R$. It follows, because $R$ is a domain, that $M$ must contain (essentially, since $M$ is uniform) a submodule isomorphic to $R$. Also, $R$ is weakly-injective (Example 1.13(ii)); therefore, by Proposition 1.7, $M$ is weakly-injective. If $M$ is torsion, then $M$ is artinian [5, Theorem 1.3]. Hence, since simple modules are injective, $M=\operatorname{Soc} M$ is injective. Our result follows from Theorem 2.5 .

3.2. COROLLARY. For a hereditary noetherian ring $R$, the following conditions are equivalent:

(i) $R$ is (right) weakly-semisimple;

(ii) every (right) $R$-module is weakly $R$-injective;

(iii) domains of injectivity in Mod- $R$ are closed under injective hulls;

(iv) $R$ is (right) $Q I$.

Proof. (i) implies (ii) is trivial. Lemma 1.8 yields that (ii) implies (iii), and Lemma 1.11 gives (iii) implies (iv). By Theorem 3.1, (iv) is equivalent to (i). 
3.3. Remark. While we do not know if the classes of right QI-rings and right weakly-semisimple rings coincide, Theorem 3.1 yields that a right QI-ring which is not right weakly-semisimple would necessarily be either a counter-example to Boyle's conjecture or not left QI. Boyle's conjecture that every right QI-ring is right hereditary and the question of whether a right QI-ring must be left QI are two of the most important open problems dealing with QI-rings.

Theorem 3.1 enables us to use some criteria developed originally by Faith [5] and extended by Kosler [11] to the question of right-left symmetry of the condition of weak-semisimplicity. Kosler's criterion says that a simple right noetherian right V-ring $R$ which satisfies the restricted right minimum condition is a left $V$-ring if and only if $R$ is left Goldie. A ring satisfies the restricted right minimum condition if $R / K$ is artinian whenever $K$ is an essential right ideal of $R$.

3.4. THEOREM. If a ring $R$ satisfies the restricted right minimum condition and is right weakly-semisimple, then $R$ is also left-weakly-semisimple.

Proof. The proof in [11, Theorem 4.2] basically shows that under restricted right minimum conditions a left Goldie right noetherian right V-ring is two-sided noetherian and hereditary. Our result follows from Example 1.13(v) and Theorem 3.1.

\section{REFERENCES}

1. J. Beachy and W. D. Blair, Rings whose faithful left ideals are cofaithful, Pacific J. Math. 58 (1975), 1-13.

2. A. K. Boyle, Hereditary QI-rings, Trans. Amer. Math. Soc. 192 (1974), 115-120.

3. A. K. Boyle and K. R. Goodearl, Rings over which certain modules are injective, Pacific J. Math. 58 (1975), 43-53.

4. A. K. Boyle, Injectives containing no proper quasi-injective submodules, Comm. Algebra 4 (1976), 775-785. $67-85$.

5. D. Eisenbud and J. C. Robson, Modules over Dedekind prime rings, J. Algebra 16 (1970),

6. C. Faith, When are proper cyclics injective? Pacific J. Math. 45 (1973), 97-111. 113-119.

7. C. Faith, On hereditary rings and Boyle's conjecture, Arch. Math. (Basel) 27 (1976),

8. J. S. Golan and Z. Papp, Cocritically nice rings and Boyle's conjecture, Comm. Algebra 8 (1980), 1775-1798.

9. R. Gordon and J. C. Robson, Krull dimension, Mem. Amer. Math. Soc. 133 (1970).

10. S. K. Jain and S. R. López-Permouth, Rings whose cyclics are essentially embeddable in projective modules, J. Algebra 128 (1990), 257-269. 467-473.

11. K. Kosler, On hereditary rings and noetherian V-rings, Pacific J. Math. 103 (1982),

12. G. O. Michler and $O$. E. Villamayor, On rings whose simple modules are injective, $J$. Algebra 25 (1973), 185-201.

13. S. Mohamed and B. J. Müller, Continuous and discrete modules, London Math. Soc. Lecture Note Series 147 (Cambridge University Press, 1990).

S. K. Jain and S. R. López-Permouth:

Department of Mathematics

OHIO UNIVERSITY

Athens, OH 45701
Surjeet Singh:

Department of Mathematics

KUWAIT UNIVERSITY

KuwaIT 